\title{
Biodegradation of phenol in a draft-tube spouted bed bioreactor with biomass attached to hydrogel particles
}

\author{
B. Safont ${ }^{1}$, A. I. Vitas ${ }^{2}$ \& F. J. Peñas ${ }^{1}$ \\ ${ }^{1}$ Department of Chemistry and Soil Science, University of Navarra, Spain \\ ${ }^{2}$ Department of Microbiology and Parasitology, \\ University of Navarra, Spain
}

\begin{abstract}
The performance of a draft-tube spouted bed bioreactor (DT-SBB) packed with hydrogel particles for biomass immobilization has been used to treat a phenolic wastewater in continuous mode. The biomass support particles were made of a cyclodextrin-based polymer and then seeded with an acclimated mixed culture. Due to the low density of such particles and also the bioreactor design, the pumping energy required to maintain a moving bed of the resulting bioparticles was very low and a recirculation flow was not necessary. The inlet phenol concentration and the phenol inlet loading rate were the operating variables studied. Phenol removal efficiency was used to monitor the process. The DTSBB showed a high removal capacity (up to $2.8 \mathrm{~kg}$-phenol $/ \mathrm{m}^{3} \mathrm{~d}$ ) with a high efficiency $(>99 \%)$. The removal capacity of the DT-SBB was found to be limited by the availability of dissolved oxygen in periods with high phenol elimination rate. The removal efficiency decreased significantly for higher loading rates $\left(>3.1 \mathrm{~kg} / \mathrm{m}^{3} \mathrm{~d}\right)$ because of phenol inhibition. The evolution of the distribution of microbial populations was also investigated. A predominance of gram-negative bacteria (especially the genera Pseudomonas and Acinetobacter) was observed during the periods of maximum degradation.
\end{abstract}

Keywords: spouted bed bioreactor, draft-tube, cyclodextrin polymer, hydrogel biomass support, phenol aerobic degradation. 


\section{Introduction}

Phenol, as a basic chemical used worldwide, is a major pollutant present in effluents from chemical process industries. Low concentrations of aqueous phenol are harmful to the environment and human health (Saha et al. [1]). Conventional methods for treating phenolic wastewaters include physicalchemical processes which lead to secondary pollution. Contrarily, biological treatments remove phenol yielding innocuous end-products. In particular, biological techniques using microorganisms attached to inert solid particles usually provide higher biomass concentrations (and therefore higher removal rates) than those achieved with suspended microorganisms. Moreover, moving bed biofilm systems such as fluidized bed bioreactors promote good heat and mass transfer characteristics. This type of reactor generally requires the recirculation of part of the effluent in order to permit the movement of bed bioparticles. On the other hand, the lighter the support material the lower the energy required for fluidization.

The main aim of this work is to study the operation of a draft-tube spouted bed bioreactor (DT-SBB) with hydrogel beads as biomass carrier particles for wastewater treatment. Phenol was chosen as the model pollutant.

The spouted bed is a fluid-solid contacting technique somehow derived from fluidized beds, but with its own characteristics. The fluid (in this case air and wastewater) enters the vessel through a spouting nozzle at the base, and flows through the bed of solid particles (Fan et al. [2]). The resulting hydrodynamic pattern shows three distinct regions: an upward jet (spout), a downward movingpacked bed (annulus) and a characteristic fountain above the bed surface (ÇeçenErbil [3], Cui and Grace [4]). Furthermore, in bioreactors, the shear forces exerted on the surface of the solids by the recirculation between the spout and annulus provides a good control of the growth of biofilm thickness (Livingston and Chase [5]). However, the use of spouted bed reactors is often limited by the existence of a maximum spoutable height or the largest height of particle bed which is possible to move in this contact regime. The inclusion of an internal draft tube solves this drawback and enhances the circulation pattern (Zhao et al. [6]).

Beads of a hydrogel material composed of $\beta$-cyclodextrin polymer (CDP) were used as biomass support particles. Since its swollen density is slightly higher than that of water, CDP beads can be fluidized with a very low energy requirement. The use of CDP as support for microorganisms has been previously reported in fluidized bed reactors (Sevillano et al. $[7,8]$ ).

\section{Material and methods}

\subsection{Experimental setup}

A schematic of the experimental system is depicted in Figure 1. A conical-base spouted bed bioreactor (diameter $51 \mathrm{~mm}$, height $0.70 \mathrm{~m}$ ) made of glass (Afora, Spain) was equipped with a PVC draft-tube (diameter $16 \mathrm{~mm}$, height $0.23 \mathrm{~m}$ ). 
The inlet spouting flow (for air, nutrients and phenol solution) was entered through a nozzle (diameter $8 \mathrm{~mm}$ ) at the conical-base of the column. A clarifier was coupled to the bioreactor outlet in order to retain the detached biomass. The aqueous phenol and nutrient solutions were separately supplied by two peristaltic pumps (Masterflex L/S, USA). No effluent recirculation flow was used. The air flow rate was regulated by a flowmeter (Aalborg, USA). The DT-SBB was operated in continuous mode at room temperature. The air flow was kept enough to assure a proper oxygen saturation level in the liquid phase. The inlet concentration and flow rate of phenol (i.e., the inlet phenol loading rate) were the operating variables considered. The substrate loading rate is defined as the phenol mass flow per unit reactor volume. The biodegradation efficiency was calculated as the relative difference between the inlet and outlet phenol loading rates.

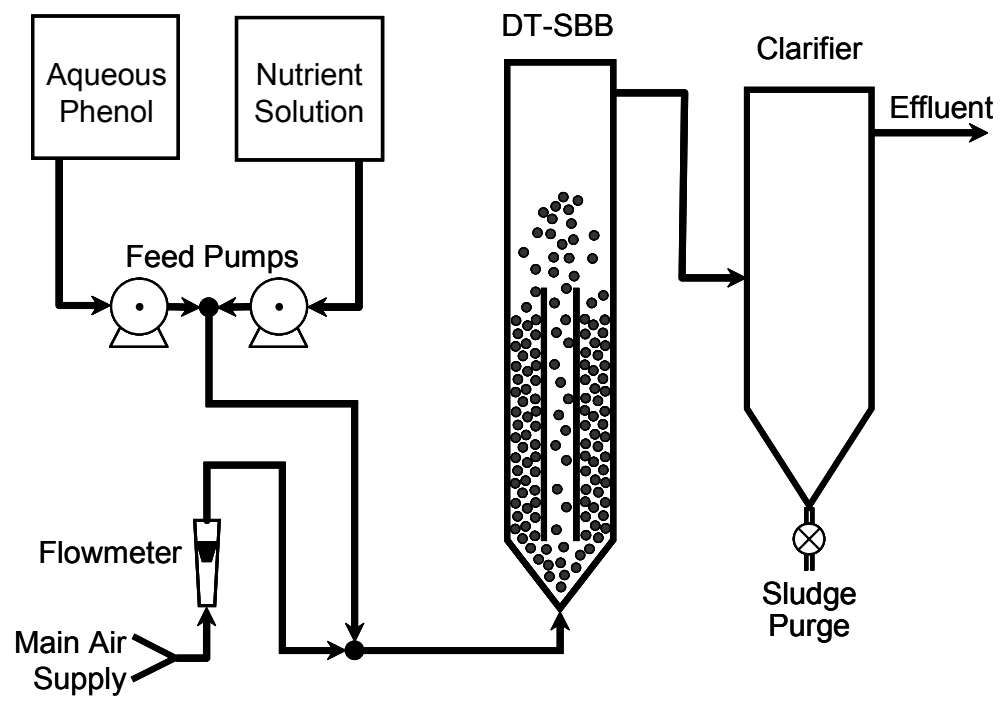

Figure 1: $\quad$ Schematic diagram of the experimental DT-SBB.

\subsection{Moving media}

Hydrogel CDP particles (nearly spherical in shape) were prepared by crosslinking $\beta$-cyclodextrin with epichlorohydrin. A detailed description can be found in Romo et al. [9]. A batch of CDP beads (bed height $19 \mathrm{~cm}$ ) with a diameter interval of $1.25-1.60 \mathrm{~mm}$ and a swollen density of $1.06 \mathrm{~g} / \mathrm{mL}$ was initially loaded into the reactor and then inoculated with an acclimated microbial mixed culture.

A synthetic wastewater with a variable phenol concentration $(2.0-2.5 \mathrm{~g} / \mathrm{L}$ in the feed tank) and equilibrated with a modified Brunner mineral medium (DSMZ Medium 457, Germany) was applied. In this case, the concentration of 
ammonium sulphate was doubled compared to the standard recipe in order to minimize nitrogen limitation conditions.

\subsection{Analytical methods}

Phenol concentration was determined by spectrophotometry (Hewlett Packard 8452, USA) at $270 \mathrm{~nm}$. Effluent samples were centrifuged (Heraeus 200, Germany) and filtered $(0.45 \mu \mathrm{m})$, according to Goswami et al. [10], just before measuring the outlet phenol concentration. Dissolved oxygen was also measured in the effluent (Hanna Instruments HI91410, Italy).

\subsection{Microbiological analysis}

Bacteria within the microbial consortium before inoculation and from the bioreactor (attached to CDP beads and suspended in broth) were isolated by plating in general and selective agar media. In order to recover the maximum number and type of microorganisms, different general agar plates have been used: yeast agar, nutrient agar and plate count agar (Biolife, Italy). Selective media used were Pseudomonas agar (cetrimide) (Oxoid, UK) and Burkholderia cepacia agar (Microkit, Spain). In addition, selective plates of yeast agar supplemented with different quantities of phenol were used to identify the most resistant strains to pollutant (Puhakka et al. [11]). To determine quantitatively the viable cells in the samples, several decimal dilutions were placed on the surface of agar plates. After incubation at $30^{\circ} \mathrm{C}$ for $24-72 \mathrm{~h}$, plates containing between 30 and 300 colonies were selected for enumeration with a colony counter (Suntex 560, Taiwan). The characterization of microbial communities was carried out by biochemical assays. Individual colonies isolated from the phenol-degrading consortium were streaked on agar plates and incubated at $30^{\circ} \mathrm{C}$ for $48 \mathrm{~h}$. The identification of microbial isolates was performed by gram staining, enzyme assays (catalase and oxidase) and API test strips (BioMerieux API 20 NE, API 50 CH, France) (Prpich and Daugulis [12]).

\section{Results and discussion}

\subsection{Biofilm growth}

The biomass growth on the hydrogel particles in the DT-SB reactor fed with aqueous phenol can be observed in Figure 2. Although not well appreciated in the $\mathrm{B} / \mathrm{W}$ photograph, the support beads were clear and transparent before microbial inoculation (day 0). Progressively, the appearance of the particles was getting darker as the solid surface was being colonized. Since the stagnant bed height scarcely increased after the whole colonization of the support media, the thickness of the growing biofilm remained quite stable throughout the study. This is a significant difference with fluidized bed bioreactors in which biofilm thickness can increase appreciably (Sevillano et al. [8]). 


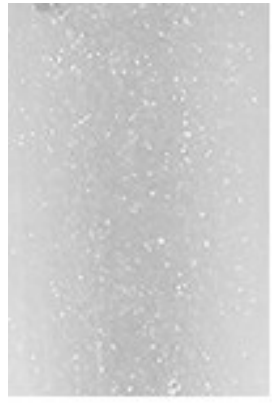

Day 0

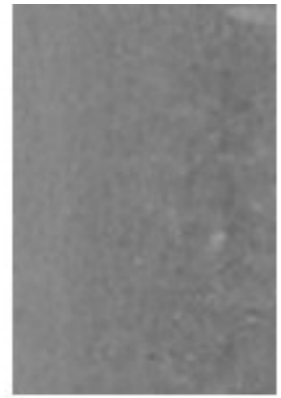

Day 53

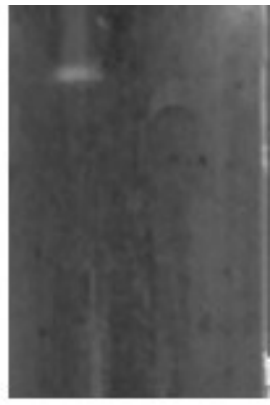

Day 79

Figure 2: $\quad$ Biofilm colonization of bed particles in the DT-SBB.

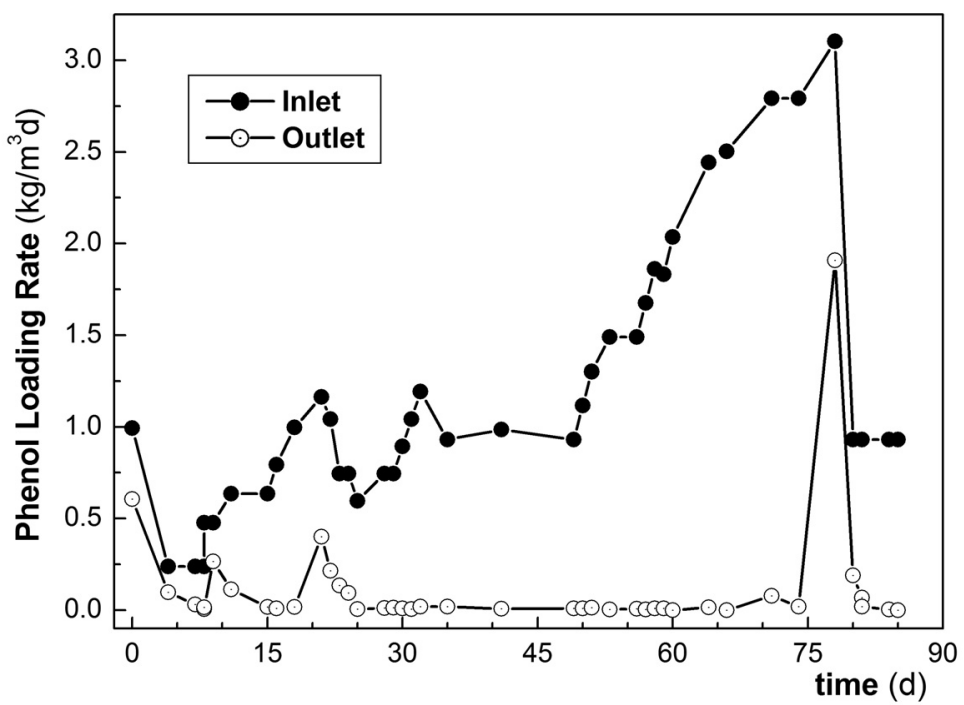

Figure 3: Phenol loading rates in the DT-SBB operation with CDP bioparticles.

\subsection{Bioreactor performance}

The reactor was operated continuously for three months. The inlet concentrations of phenol and nutrient solutions were deliberately kept high, and low the respective flow rates, in order to extend the feed supply capacity. Thereby, the values of hydraulic residence time were notably high (8-32 h) compared to those of real effluents, although the inlet substrate loading rate was as high as $3.1 \mathrm{~kg}$ $\mathrm{phenol} / \mathrm{m}^{3} \mathrm{~d}$. The organic loading rates in the influent and effluent streams are 
shown in Figure 3, whereas the outlet phenol concentration and the removal efficiency of the DT-SBB are plotted in Figure 4.

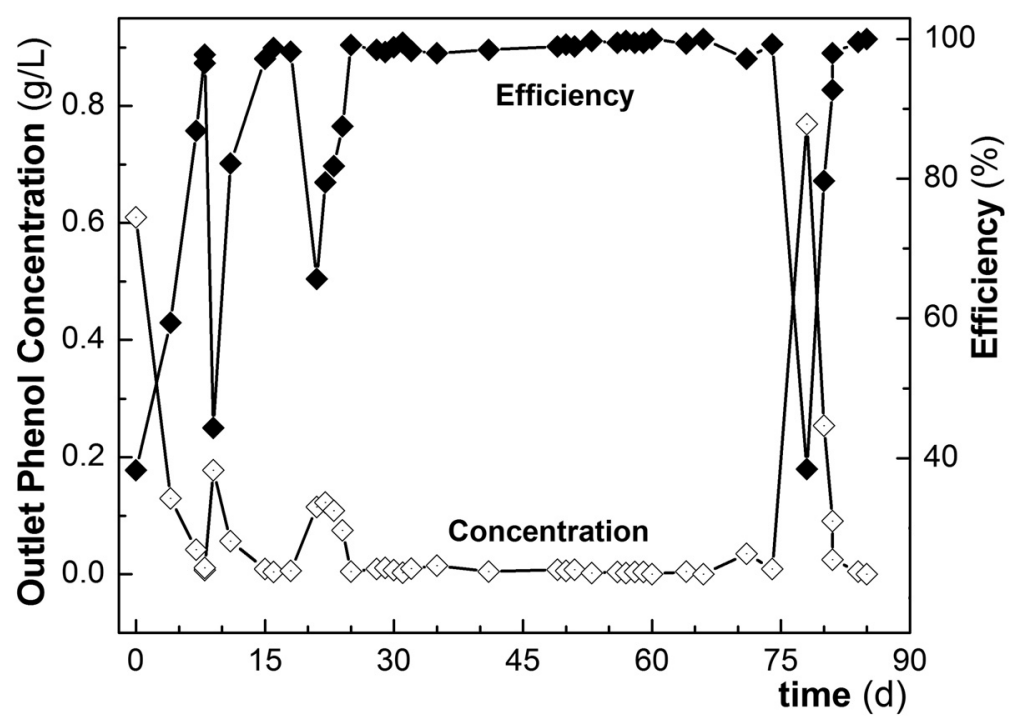

Figure 4: Outlet phenol concentration and elimination efficiency in the DTSBB.

As can be observed, the DT-SBB worked with low inlet loading rate of substrate at the starting-up in order to promote the colonization of the support particles by microorganisms. At the end of the initial acclimation period, the system was treating about $1.0 \mathrm{~kg}$-phenol $/ \mathrm{m}^{3} \mathrm{~d}$ with a high removal efficiency $(>95 \%)$. However, at the beginning of this acclimation period, the inlet load was so high that the system became oversaturated and high values of dissolved oxygen were reached.

From day 50 and on, the pollutant loading rate entering the bioreactor was progressively increased up to very high values while keeping the conversion efficiency high. This good bioreactor performance was maintained for nearly one month (until day 75). The maximum elimination capacity was achieved in the last week of this period (from day 70 to day 75 ) with $2.79 \mathrm{~kg}-\mathrm{phenol} / \mathrm{m}^{3} \mathrm{~d}$ and a removal efficiency higher than $99 \%$ (i.e., a phenol concentration of $19 \mathrm{mg} / \mathrm{L}$ in effluent).

By day 80 , when the inlet loading rate had been raised up to $3.10 \mathrm{~kg}$ phenol $/ \mathrm{m}^{3} \mathrm{~d}$, the removal capacity of the system was saturated and the removal efficiency decreased significantly (below 40\%) with the outlet phenol concentration increasing up to $0.77 \mathrm{~g} / \mathrm{L}$. As expected, the stronger the substrate inhibition the higher the phenol concentration in the effluent. This behavior is characteristic of the metabolism of toxic compounds, such as was observed by Watanabe et al. [13] in an activated sludge process when the inlet phenol 
concentration exceeded the upper limit of $2.0 \mathrm{~kg} / \mathrm{m}^{3} \mathrm{~d}$. Several factors could explain the difference in the maximum biodegradable phenol load between that reported and that determined in the present work. First, the DT-SBB is a fixed biofilm reactor and the other was a suspended biomass system, and it is wellknown the larger resistance of the former compared to the latter (Sá and Boaventura [14]). Furthermore, the use of a hydrogel support with proven sorption ability towards phenol (Romo et al. [15]) would enhance the protection of the biofilm from shock-loading (Sevillano et al. [8]). After the saturation point was reached, as the phenol removal capacity dropped abruptly, the inlet load was reduced to $0.93 \mathrm{~kg} / \mathrm{m}^{3} \mathrm{~d}$ in order to recover the process efficiency.

On the one hand, dissolved oxygen (DO) could have been a limiting factor in the DT-SBB performance. The DO concentration as measured in the DT-SBB effluent is given in Figure 5. An air flow rate range of 5.4-13.8 L/h was applied. The maximum air flow entering the bioreactor was limited because of the risk of elutriation of bed particles. The fluctuations in the DO concentration during the first three weeks were due to the decoupling between aeration and biological activity during the acclimation period. High values of DO concentration would be related to bad working conditions with poor degradation activity, whereas low DO values would be associated with high elimination capacity. Nevertheless, very low DO levels (close to zero) with high substrate removal rates would be showing a problem of oxygen mass transfer. Therefore, the improvement of the aeration system can play an important role in the enhancement of the DT-SBB performance.

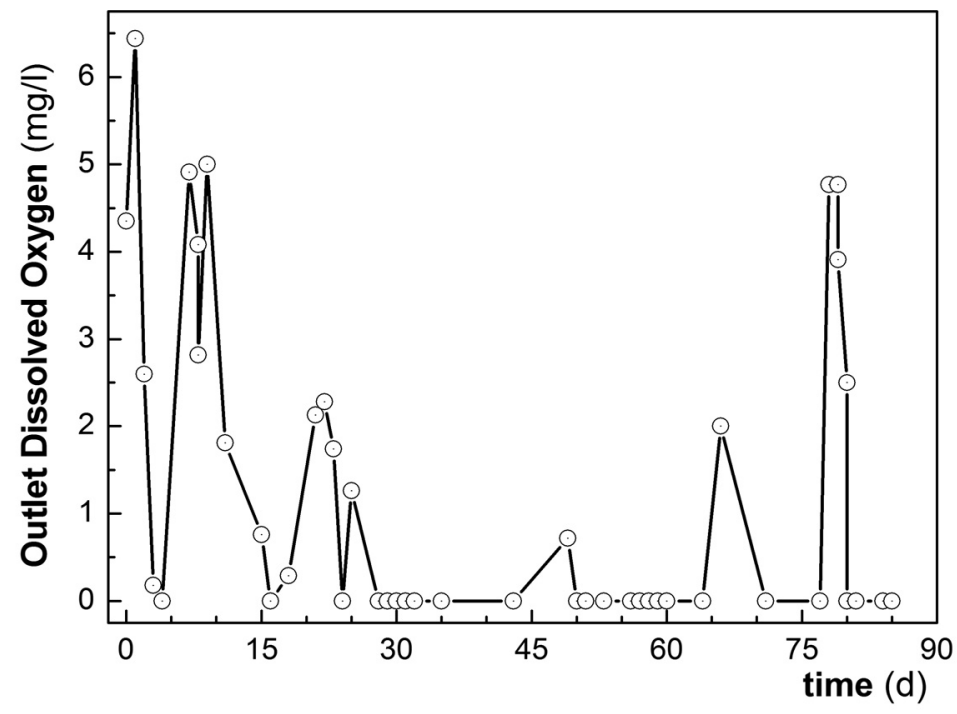

Figure 5: Dissolved oxygen profile in the DT-SBB effluent. 


\subsection{Microbiological studies}

Table 1 shows the isolated microorganisms during the phenol adaptation phase before being inoculated into the DT-SBB. The predominant species present in the microbial consortium included both gram-positive and gram-negative bacteria, being the genus Bacillus the most abundant (about $80 \%$ of total).

Table 1: Identified microorganisms in the inoculum during the phenol acclimation period.

\begin{tabular}{lcl}
\hline \multicolumn{1}{c}{ Isolate } & $\begin{array}{c}\text { Concentration } \\
\text { (CFU/mL) }\end{array}$ & Characteristics \\
\hline Bacillus circulans 1 & $4.6 \times 10^{5}$ & Gram-positive, catalase-positive \\
Burkholderia cepacia & $6.7 \times 10^{4}$ & Gram-negative, oxidase-positive \\
Bacillus thuringiensis & $5.0 \times 10^{4}$ & Gram-positive, catalase-positive \\
Ochrobactrum anthropi & $2.3 \times 10^{3}$ & Gram-negative, oxidase-positive \\
Alcaligenes xilosoxidans & $1.1 \times 10^{3}$ & Gram-negative, oxidase-positive \\
\hline
\end{tabular}

The identification and enumeration of the microbial consortium within operational phase of the DT-SBB showed a strong selection of gram-negative bacteria, where genera Comamonas and Acinetobacter appeared to be the predominant (Table 2). As shown in Figure 3, the increase of the inlet loading rate of phenol, together with the hydrodynamic conditions of the DT-SBB, has a marked selective effect on the microbial community. None of the gram-positive bacteria identified in the acclimation period were isolated during the bioreactor operation.

Table 2: $\quad$ Identified microorganisms in DT-SBB samples.

\begin{tabular}{lcc}
\hline \multicolumn{1}{c}{ Isolate } & $\begin{array}{c}\text { Concentration } \\
\text { (CFU/mL) }\end{array}$ & Characteristics \\
\hline Comamonas acidovorans & $2.1 \times 10^{7}$ & Gram-negative, oxidase-positive \\
Acinetobacter baummanni & $1.2 \times 10^{7}$ & Gram-negative, oxidase-positive \\
Ochrobactrum anthropi & $8.0 \times 10^{6}$ & Gram-negative, oxidase-positive \\
Burkholderia cepacia & $6.0 \times 10^{6}$ & Gram-negative, oxidase-positive \\
\hline
\end{tabular}

\section{Conclusions}

In conclusion, hydrogel particles of a cyclodextrin-based polymer have been shown to be an effective support medium for attached biomass in a draft-tube spouted bed bioreactor treating a phenolic wastewater. The shear forces caused by the spouting hydrodynamics on the bed particles exert a good control of biofilm growth while maintaining an efficient phenol biodegradation. In addition, since no water recirculation flow was necessary to move the bed due to the low support density, the DT-SBB operational costs should be smaller than 
those of conventional fluidized bed bioreactors. Experimental results showed that this system could be used to satisfactorily treat effluents containing high phenol inlet loading rates (up to $2.8 \mathrm{~kg} / \mathrm{m}^{3} \mathrm{~d}$ ) with high removal efficiency $(>99 \%)$. Pseudomonas and Acinetobacter were the predominant genera in optimal conditions of DT-SBB performance. Despite the high phenol removal efficiency achieved, more research is needed to improve the aeration efficiency in order to avoid oxygen-limiting conditions as well as any changes in microbial community by substrate inhibition.

\section{Acknowledgements}

The authors wish to thank the Government of Navarre (Department of Education) for providing the funding for this work.

\section{References}

[1] Saha, N.C., Bhunia, F. \& Kaviraj, A., Toxicity of phenol to fish and aquatic ecosystems, Bulletin of Environmental Contamination and Toxicology, 63(2), pp. 195-202, 1999.

[2] Fan, L.S., Hwang, S.J. \& Matsuura, A., Hydrodynamic behaviour of a draft tube gas-liquid-solid spouted bed, Chemical Engineering Science, 39(12), pp. 1677-1688, 1984.

[3] Çecen-Erbil, A. Effect of the annulus aeration on annulus leakage and particle circulation in a three-phase spout-fluid bed with a draft tube, Powder Technology, 162(1), pp. 38-49, 2006.

[4] Cui, H. \& Grace, J.R., Spouting of biomass particles: A review, Bioresource Technology, 99(10), pp. 4008-4020, 2008.

[5] Livingstone, A. \& Chase, H., Development of a phenol degrading fluidized bed bioreactor for constant biomass holdup, Chemical Engineering Journal, 45(3), pp. B31-B47, 1991.

[6] Zhao, X.L., Yao, Q. \& Li, S.Q., Effects of draft tubes on particles velocity profiles in spouted beds, Chemical Engineering \& Technology, 29(7), pp. 875-881, 2006.

[7] Sevillano, X., Romo, A., Isasi, J.R., González-Gaitano, G. \& Peñas, J., Start-up of a fluidized bed bioreactor with a $\beta$-cyclodextrin support for treating wastewater. Waste Management and the Environment, eds. D. Almorza et al., WIT Press: Southampton, pp. 709-715, 2002.

[8] Sevillano, X., Romo, A., Isasi, J.R. \& Peñas, F.J., Feasibility study of degradation of phenol in a fluidized bed bioreactor with a cyclodextrin polymer as biofilm carrier, Biodegradation, 19(4), 589-597, 2008.

[9] Romo, A., Peñas, F.J., Sevillano, X. \& Isasi, J.R., Application of factorial experimental design to the study of the suspension polymerization of $\beta$ cyclodextrin and epichlorohydrin. Journal of Applied Polymer Science, 100(4), 3393-3402, 2006.

[10] Goswami, M., Shivaraman N. \& Singh, R.P., Microbial metabolism of 2chlorophenol, phenol and p-cresol by Rhodococcus erythropolis M1 in 
coculture with Pseudomonas fluorescens P. Microbiological Research, 160(2), pp. 101-109, 2005.

[11] Puhakka, J.A., Herwig R.P., Koro P.M., Wolfe, G.V. \& Ferguson J.F., Biodegradation of chlorophenols by mixed and pure cultures from a fluidized-bed reactor. Applied Microbiology and Biotechnology, 42(6), pp. 951-957, 1995.

[12] Prpich, G.P. \& Daugulis, A.J., Enhanced biodegradation of phenol by a microbial consortium in a solid-liquid two phase partitioning bioreactor. Biodegradation, 16(4), pp. 329-339, 2005.

[13] Watanabe, K., Hino, S. \& Takahashi, N., Responses of activated sludge to an increase in phenol loading, Journal of Fermentation Bioengineering, 82(5), pp. 522-524, 1996.

[14] Sá, C.S.A. \& Boaventura, R.A.R., Biodegradation of phenol by Pseudomonas putida DSM 548 in a trickling bed reactor, Biochemical Engineering Journal, 9(3), pp. 211-219, 2001.

[15] Romo, A., Peñas, F.J., Sevillano, X., Isasi, J.R., García-Zubiri, I.X. \& González-Gaitano, G., Extraction of phenols from aqueous solutions by $\beta$ cyclodextrin polymers. Comparison of sorptive capacities with other sorbents, Reactive \& Functional Polymers, 68(1), pp. 406-413, 2008. 